Human Services, National Institute for Occupational Safety and Health; September 1991 (NIOSH publication no. 91-119): appendix H.

2) Guide to Industrial HEPA-Filtered Respirator Protection. Morgantown, WV: U.S. Department of Health and Human Services, National Institute for Occupational Safety and Health; September 1987 (NIOSH publication no. 87-116).

3) The National Institute for Occupational Safety and Health (NIOSH). Physicians there may assist with questions regarding use of the HEPA respirator. Call (800) 3564674 between 1:00 PM and 4:00 PM Eastern Standard Time. FAX (513) 533-8573.

4) The Occupational Safety and Health Administration's local or regional office for your region; or in delegated states, the delegated state agency.

5) Centers for Disease Control and Prevention. A phone line has been established to answer any questions about TB. Call (404) 639-8120.

6) The American Hospital Association (AI-IA) offers an excellent TB resource packet (catalog no. 094692) for $\$ 50$ ( $\$ 15$ for members), plus shipping; call (800) 242-2626. Copies of the proceedings of a 4-hour preconference seminar held at the AHA's National Symposium on Health Care Safety and the Environ- ment in February 1993 are available; call (312) 2804496.

OSHA issued the HEPA respirator mandate for the same reason it issues all of its rules: to protect workers from job-related injury. It is expected that OSHA will modify its mandates, based on the CDC's final guidelines. In addition, NIOSH is recertifying respirators and is adding several other respirators to the current list.

Meanwhile, hospitals are forced to use their limited funds to buy equipment and establish protocols without a consensus from regulatory agencies. Until regulations are changed, we risk investing precious healthcare dollars in policies and protocols that may be unnecessary. Clearly, we must work together to find a better way to keep order.

\section{REFERENCES}

1. Centers for Disease Control and Prevention. Draft guidelines for preventing the transmission of tuberculosis in health-care facilities, second edition: notice of comment period. Federal Register 1993;58:52810-52854.

2. Pugliese G. TB respirators: what's required and what isn't. Material Management 1994;3:30-36.

3. American Health Consultants. AHA, SHEA question cost, scope of CDC draft TB guidelines. Hosp Infect Control 1994;21:35.

\title{
Limited Supplies of Inactivated Poliovirus Vaccine in US
}

by Gina Pugliese, RN, MS Medical News Editor

The CDC reports a shortage of inactivated poliovirus vaccine (IPV) in the United States. The Food and Drug Administration, the manufacturers, and the distributors are working to resolve this shortage.

Until IPV becomes readily availa- ble, $\mathrm{CDC}$ recommends that its use be restricted to: (1) never-vaccinated persons aged $>18$ years who are at risk for exposure to wild poliovirus (eg, who will be traveling to areas in which poliomyelitis is endemic), and (2) persons for whom oral polio vaccine (OPV) is contraindicated (ie, persons diagnosed with or living in a household with a person with a congenital or acquired immune deficiency). Inadequately or fully vaccinated adults who have previously received IPV or OPV and need poliovirus vaccine can be given OPV.

Information about obtaining IPV for high-risk persons is available from the distributor, Connaught Laboratories, Inc, (800) 822-2463. 